\title{
THE
}

\section{Predictors of Mortality Among U.S. Veterans With Streptococcus Pneumoniae Infections}

Jacob B. Morton

University of Rhode Island

\section{J. Morrill}

University of Rhode Island

Kerry L. LaPlante

University of Rhode Island, kerrylaplante@uri.edu

Aisling R. Caffrey

University of Rhode Island, aisling_caffrey@uri.edu

Follow this and additional works at: https://digitalcommons.uri.edu/php_facpubs

\section{The University of Rhode Island Faculty have made this article openly available.}

Please let us know how Open Access to this research benefits you.

This is a pre-publication author manuscript of the final, published article.

Terms of Use

This article is made available under the terms and conditions applicable towards Open Access Policy Articles, as set forth in our Terms of Use.

\section{Citation/Publisher Attribution}

Morton, J. B., Morrill, H. J., LaPlante, K. L., \& Caffrey, A. R. (2016). Predictors of Mortality Among U.S. Veterans With Streptococcus Pneumoniae Infections. American Journal of Preventive Medicine, 52(6), 769-777. doi: 10.1016/j.amepre.2016.10.023

Available at: http://dx.doi.org/10.1016/j.amepre.2016.10.023 
Predictors of Mortality Among U.S. Veterans With Streptococcus Pneumoniae Infections

Jacob B. Morton, PharmD, MBA, BCPS,${ }^{1,2}$ Haley J. Morrill, PharmD, ${ }^{1,2}$ Kerry L. LaPlante, PharmD, ${ }^{1,2,3}$ Aisling R. Caffrey, PhD, $\mathrm{MS}^{1,2,4}$

From the ${ }^{1}$ Veterans Affairs Medical Center, Infectious Diseases Research Program and Center of Innovation in Long Term Services and Supports, Providence, Rhode Island; ${ }^{2}$ Department of Pharmacy Practice, College of Pharmacy, University of Rhode Island, Kingston, Rhode Island; ${ }^{3}$ Division of Infectious Diseases, Warren Alpert Medical School of Brown University, Providence, Rhode Island; and ${ }^{4}$ Brown University School of Public Health, Providence, Rhode Island

Address correspondence to: Aisling R. Caffrey, PhD, MS, Assistant Professor, University of Rhode Island, 7 Greenhouse Road, Kingston RI 02881. E-mail: aisling_caffrey@ uri.edu. 
Introduction: Serious Streptococcus pneumoniae infections, encompassing pneumonia, bacteremia, and meningitis, are a major cause of mortality. However, literature regarding mortality is often limited to invasive pneumococcal disease, excluding pneumonia. This study sought to identify predictors of mortality among adults with serious pneumococcal disease, including pneumonia and invasive pneumococcal disease.

Methods: This was a nested case-control study of unvaccinated older Veterans with positive $S$. pneumoniae cultures (blood, cerebrospinal fluid, respiratory) admitted to Veterans Affairs medical centers nationally between 2002 and 2011. Patients vaccinated against pneumococcal disease were excluded. Using multivariable logistic regression, predictors of 30-day mortality were identified, including patient demographics, comorbidities during admission, and medical history within the previous year.

Results: Among 9,468 patients, there were 9,730 serious pneumococcal infections; 1,764 (18.6\%) resulted in death within 30 days (cases), whereas 7,966 did not (controls). Pneumonia accounted for half $(49.4 \%, n=871)$ of all deaths. Mortality predictors consistent with vaccine recommendations included dialysis (during hospitalization, $\mathrm{OR}=3.35,95 \% \mathrm{CI}=2.37,4.72$ ), moderate to severe liver disease (during hospitalization, $\mathrm{OR}=2.47,95 \% \mathrm{CI}=1.53,3.99$; within 1 year, $\mathrm{OR}=1.49,95 \% \mathrm{CI}=1.01,2.20$ ), and neutropenia (during hospitalization, $\mathrm{OR}=2.67,95 \%$ $\mathrm{CI}=1.32,5.42$ ). Predictors not included in current recommendations included dementia (during hospitalization, $\mathrm{OR}=1.8,95 \% \mathrm{CI}=1.23,2.61)$ and neurologic disorders (during hospitalization, $\mathrm{OR}=1.86,95 \% \mathrm{CI}=1.42,2.45$; within 1 year, $\mathrm{OR}=1.28,95 \% \mathrm{CI}=1.02,1.59)$.

Conclusions: Several mortality predictors among unvaccinated Veterans with serious pneumococcal disease were consistent with pneumococcal vaccine recommendations, including 
organ or immune system dysfunction-related conditions. Other predictors, including neurologic disorders or dementia, may warrant expanded vaccination recommendations. 


\section{INTRODUCTION}

Streptococcus pneumoniae is a leading cause of morbidity and mortality worldwide, much of which is the result of serious pneumococcal infections, including pneumonia, bacteremia, and meningitis. ${ }^{1,2}$ Although invasive pneumococcal disease, which encompasses bacteremia and meningitis, results in more than 15,000 hospitalizations and a mortality of at least $15 \%$, much of the burden of serious pneumococcal disease is related to pneumonia. ${ }^{2}$ In the U.S. alone, an estimated 900,000 individuals develop pneumococcal pneumonia annually, resulting in approximately 400,000 hospitalizations. ${ }^{1-3}$ Though mortality for pneumococcal pneumonia is only $5 \%-7 \%$ overall, this includes less severe pneumonias managed in the outpatient setting. ${ }^{5}$ Mortality in adults aged 65 years and older and those requiring hospitalization is approximately $15 \%$, as these individuals may be more susceptible to complications, including development of bacteremia. $^{1-4}$

Despite the burden of pneumococcal pneumonia, studies assessing predictors of mortality associated with serious pneumococcal disease have focused primarily on patients with invasive pneumococcal disease only, excluding patients with nonbacteremic pneumonia. ${ }^{1,2}$ As such, the purpose of this study was to identify independent predictors of mortality among unvaccinated adults with serious pneumococcal disease, including pneumonia and invasive pneumococcal disease.

\section{METHODS}

The IRB and the Research and Development Committee of the Providence Veterans Affairs Medical Center approved this study. 
The Veterans Health Administration is the largest healthcare system in the U.S., composed of 152 medical centers along with 1,400 outpatient facilities and community living centers. ${ }^{10}$ The electronic medical record utilized by the Veterans Health Administration captures both inpatient and outpatient visits dating back to 1999. The Veterans Affairs databases have been described previously. ${ }^{11,12}$ An analytic database was created from numerous Veterans Affairs databases capturing patient care, including demographics, inpatient stays, outpatient visits, pharmacy dispensings and administrations, health factors, surgeries and procedures, laboratory and microbiology results, immunizations, and vital status.

\section{Study Population}

This was a national nested case-control study of Veterans aged $\geq 50$ years requiring inpatient care for serious pneumococcal disease (bacteremia, meningitis, pneumonia) between January 1, 2002 and December 31, 2011. ${ }^{11}$ Adults aged $\geq 50$ years were selected as they are at the highest risk of developing these serious pneumococcal infections. ${ }^{5}$ Positive $S$. pneumoniae sputum, blood, and cerebrospinal fluid cultures were identified using microbiology datas. ${ }^{11}$ Pneumonia was identified by positive sputum culture in addition to a pneumonia ICD-9 diagnosis code. From this cohort, individuals who had not received the pneumococcal vaccine, as determined from pharmacy, procedure, and immunization status data, within 5 years of the positive culture were selected for inclusion.

Cases were defined as those who died from any cause within 30 days of positive culture, and controls were those alive at 30 days. All available controls were included as mortality was expected to be approximately $20 \%$, resulting in a 1:4 ratio of cases to controls, and matching was not utilized as all demographic and clinical characteristics were assessed as predictors. ${ }^{13,14}$ Using 
ICD-9 and procedure codes, current comorbidities and medical history were determined, and defined as the presence of disease states during the index admission and within 1 year of the positive culture date, respectively. Use of immunosuppressant medications was also assessed using pharmacy data. Immunosuppressant medication use was defined as corticosteroid, monoclonal antibody, or antineoplastic agent use within 30 days prior to positive culture date.

Potential predictors of mortality included patient demographics, disease states present during the admission as well as those documented within 1 year prior to positive culture, the presence of immunosuppressant medications, presence of invasive disease, and length of stay. All current Advisory Committee on Immunization Practices (ACIP) recommendations for vaccination, such as age, immunodeficiency, diabetes, chronic lung disease, chronic heart disease, liver disease, and alcoholism, were included as potential predictors in order to quantify risk of mortality to unvaccinated individuals with these conditions.

\section{Statistical Analysis}

Independent predictors of 30-day all-cause mortality were identified utilizing a backward manual stepwise logistic regression model. ${ }^{14}$ As part of the univariate analysis, variables were deemed to be included in the subsequent multivariable analysis at a $p$-value $<0.10 .{ }^{14}$ Significant variables from the univariate analysis were then removed in a stepwise fashion from the multivariable model until all remaining variables within the model demonstrated a Wald $p$-value $<0.05 .^{14}$ Variables in the predictor model were assessed for multicollinearity. All statistical analyses were performed with SAS, version 9.2.

\section{RESULTS}


There were 14,511 episodes of serious pneumococcal infections, of which 4,781 (33\%) episodes involved those who had received the pneumococcal vaccine in the previous 5 years and were therefore excluded. Of the 9,468 identified unvaccinated patients, there were 9,730 episodes of serious pneumococcal infections; 1,764 (18.6\%) died within 30 days of culture (cases) and 7,966 did not die (controls). Pneumonia $(62 \%, n=6,075)$, bacteremia $(26 \%, n=2,554)$, and bacteremic pneumonia $(11 \%, n=1,060)$ were the most common infections, with a 30 -day mortality rate of $14 \%, 23 \%$, and $29 \%$, respectively. A total of 41 episodes $(0.4 \%)$ of pneumococcal meningitis occurred during the study period. Approximately half $(50.6 \%, n=893)$ of those who died within 30 days had invasive pneumococcal disease. Overall 30-day mortality in patients with invasive disease was $24 \%$. Characteristics of cases and controls are described in detail in Table 1. Compared with those alive 30 days after positive culture, those who died within 30 days were significantly $(p<0.05)$ older (mean age, $71[\mathrm{SD}=11]$ years vs $67[\mathrm{SD}=11]$ years) and, in the previous year, were significantly more likely to have a history of infections, including pneumococcal infections (38.9\% vs $28.3 \%$ ), pneumonia (35.4\% vs $24 \%$ ), bacteremia (6.2\% vs $2.7 \%)$, urinary tract infections $(15.1 \%$ vs $7.3 \%)$, and skin or soft tissue infections (15.9\% vs 10.7\%). Several comorbidities present during the index admission were significantly $(p<0.05)$ more common among cases compared with controls, including heart failure (18.9\% vs $13.1 \%)$, cardiac arrhythmias (28.5\% vs $18.8 \%)$, chronic respiratory disease (32.8\% vs $39 \%)$, chronic renal disease $(11.1 \%$ vs $7.3 \%)$, mild hepatic disease $(7.9 \%$ vs $5.1 \%)$, moderate or severe hepatic disease (3.2\% vs $0.8 \%$ ), and dialysis ( $5.9 \%$ vs $1.9 \%)$. Additionally, cases were significantly more likely to have received an immunocompromising medication within 30 days of admission (28.2\% vs $22.5 \%)$ and have a history of chronic conditions, including heart failure $(27.7 \%$ vs $16.6 \%)$, coronary artery disease $(34.6 \%$ vs $25.1 \%)$, chronic respiratory disease $(51.6 \%$ vs 
$45.3 \%$ ), diabetes mellitus ( $27 \%$ vs $21.5 \%$ ), or an immunocompromising condition (42.3\% vs $31.8 \%)$.

Based on the multivariable regression analysis, predictors of 30-day all-cause mortality present within 1 year of culture that are also current ACIP recommendations for pneumococcal vaccination included metastatic malignancy (Table 2; OR 1.53), mild hepatic disease (OR=1.35), moderate to severe hepatic disease $(\mathrm{OR}=1.49)$, and myocardial infarction $(\mathrm{OR}=1.31)$. Significant predictors of mortality present during hospitalization that are also current pneumococcal vaccination indications included age $(\mathrm{OR}=1.03)$, chronic heart failure $(\mathrm{OR}=1.5)$, corticosteroid use within 30 days of positive culture $(\mathrm{OR}=1.3)$, dialysis $(\mathrm{OR}=3.35)$, metastatic malignancy $(\mathrm{OR}=2.54)$ and non-metastatic malignancy $(\mathrm{OR}=2.34)$, mild hepatic disease $(\mathrm{OR}=1.63)$ and moderate to severe hepatic disease $(\mathrm{OR}=2.47)$, myocardial infarction $(\mathrm{OR}=1.75)$, and neutropenia $(\mathrm{OR}=2.67)$.

Significant predictors of mortality present within 1 year of culture that are not ACIP vaccination recommendations included cerebrovascular disease (Table 2; $\mathrm{OR}=1.25$ ), chronic pressure ulcers $(\mathrm{OR}=2.04)$, Elixhauser comorbidity score $(\mathrm{OR}=1.16)$, fluid or electrolyte disorders $(\mathrm{OR}=1.20)$, Gram-negative infections (OR=1.48), methicillin-resistant Staphylococcus aureus (MRSA) infections $(\mathrm{OR}=1.59)$, neurologic disorders $(\mathrm{OR}=1.28)$, and weight loss $(\mathrm{OR}=1.21)$. Significant predictors present during hospitalization included cardiac arrhythmias $(\mathrm{OR}=1.41)$, chronic pressure ulcers $(\mathrm{OR}=1.42)$, coagulopathies $(\mathrm{OR}=2.30)$, dementia $(\mathrm{OR}=1.80)$, endocarditis $(\mathrm{OR}=2.74)$, fluid or electrolyte disorders $(\mathrm{OR}=1.69)$, neurologic disorders $(\mathrm{OR}=1.86)$, and vancomycin-resistant Enterococcus species infection (OR=3.78). 


\section{DISCUSSION}

This study identified independent predictors of 30-day all-cause mortality among unvaccinated adults aged 50 years and older with serious pneumococcal disease. Current literature related to mortality among patients with pneumococcal infections is primarily limited to patients with invasive pneumococcal disease, excluding nonbacteremic pneumonia. ${ }^{15-23}$ Furthermore, several of these studies' cohorts excluded immunocompetent patients. ${ }^{21-23}$ Based on currently available literature, this study is the first to provide insight into predictors of mortality in a large real-world sample of patients, in particular older Veterans, with invasive and non-invasive pneumococcal disease.

Risk of developing pneumococcal disease is highest in adults aged 65 years and older, immunocompromised individuals, and those with chronic illnesses, including diabetes, chronic lung disease, chronic heart disease, liver disease, and alcoholism. ${ }^{24}$ As such, ACIP recommends that these populations be vaccinated against pneumococcal disease. Pneumococcal vaccination prevents incident infection and, as such, has led to a reduction in negative infection-related outcomes, including mortality. ${ }^{25-27}$

Despite the established benefits of pneumococcal vaccination, multiple studies have identified that current pneumococcal vaccination rates are far below the Healthy People 2020 goals. ${ }^{11,28}$ In 2013, the National Health Interview Survey indicated that approximately 67 million at-risk individuals in the U.S. have not received pneumococcal vaccination, placing them at an increased risk of worse outcomes and more severe pneumococcal infection. ${ }^{5,29}$ This is 
concerning, as previous research has demonstrated that people with ACIP indications for pneumococcal vaccination are twice as likely to die as those without indications if they develop invasive pneumococcal disease. ${ }^{30}$

Not surprisingly, several $(41 \%, 14 / 34)$ of the predictors of mortality identified are consistent with conditions that also increase the risk for development of invasive pneumococcal disease, and thus support current ACIP recommendations for the vaccination of adults against pneumococcal disease. ${ }^{31-33}$ Although pneumococcal vaccination is primarily intended to prevent development of disease, a recent study demonstrated that implementation of programs to increase vaccination with both the 23 -valent polysaccharide pneumococcal vaccine and the 13 valent pneumococcal conjugate vaccine have resulted in not only decreased incidence of invasive pneumococcal disease but also mortality. ${ }^{26}$ Vaccination strategies utilizing both vaccines may minimize negative outcomes, including mortality.

In addition to conditions already identified by ACIP, this study also revealed several other predictors of mortality that are not current recommendations for pneumococcal vaccination. These included neurologic disorders, fluid or electrolyte disorders, chronic pressure ulcers, and MRSA or Gram-negative infections. In particular, patients with neurologic disorders, including seizure disorders, multiple sclerosis, Parkinson disease, and ataxia, warrant consideration. A recent literature review demonstrated that epilepsy, Parkinson disease, and multiple sclerosis were all risk factors for the development of community-acquired pneumonia, increasing risk by twofold to fourfold. ${ }^{34}$ Neurologic disorders are believed to increase the risk of developing pneumococcal disease owing to a higher likelihood of aspiration. ${ }^{2}$ As neurologic disorders were 
also independent predictors of mortality in this study, and considering the infection and potential mortality prevention afforded by pneumococcal vaccination, further studies assessing the impact of pneumococcal vaccination on outcomes in patients with a history of neurologic disorders are warranted. ${ }^{25-27,33}$

Fluid and electrolyte disorders, chronic pressure ulcers, and MRSA or Gram-negative infections were also predictive of mortality. Fluid and electrolyte disorders, which may also be related to renal dysfunction, were predictive both during hospitalization as well as within 1 year of culture. Other studies have previously demonstrated the risk of mortality associated with renal impairment of all severities. ${ }^{35,36}$ However, upon further analysis of the results of this study, diagnosis codes for renal dysfunction were present less than $20 \%$ of the time when fluid and electrolyte disorders were also present. Thus, mortality in patients with fluid and electrolyte disorders may be linked to conditions not related to renal impairment, although determining these associations is difficult solely based on diagnosis codes. More research regarding fluid and electrolyte disorders and pneumococcal disease outcomes is needed.

Chronic pressure ulcers, though predictive of mortality in this study, have also been shown to be predictive of mortality regardless of pneumococcal infection. ${ }^{37}$ A study of 258 hospitalized patients demonstrated that risk of death more than tripled in the setting of pressure ulcers. ${ }^{37}$ As such, the findings from this study of increased mortality in patients with pressure ulcers likely demonstrate the frail nature of older patients. With regard to history of either MRSA or Gramnegative infections, a plausible association with mortality is difficult to establish, as these diagnosis codes encompassed infections of all sources and severities. 
This study also demonstrated that dementia was predictive of mortality. Dementia has been shown to increase risk of developing pneumococcal infection as well as increased disease severity, likely due to an increased risk of aspiration similar to neurologic disorders. ${ }^{2,34,38} \mathrm{~A}$ retrospective study of more than 1,000 patients with a mean age of 64 years showed that dementia was an independent predictor of increased pneumococcal infection severity $(\mathrm{OR}=4.23$, $95 \% \mathrm{CI}=1.24,14.4$ ), but was not a significant predictor of mortality. ${ }^{38}$ However, severe infection, which was assessed in the aforementioned study as well as in this study via invasive disease and intensive care unit treatment, was predictive of mortality. Although dementia was only significant in the predictive model during hospitalization, the results of this study support the already identified association between dementia and poor outcomes in the setting of pneumococcal infection. Furthermore, at least $4 \%$ of patient's with Alzheimer dementia, the most common type of dementia, are younger than age 65 years, and therefore not covered by the ACIP vaccination recommendation for individuals aged 65 years and older. ${ }^{39}$ Thus, pneumococcal vaccination may minimize poor outcomes and prevent infection in this at-risk population; however, further research is warranted.

The strongest and most prevalent predictors of mortality were those consistent with organ dysfunction or immunodeficiency during the admission. Predictors that were present within 1 year of the infection appear to have an attenuated effect on mortality in relation to those predictors present during the index admission. Examples of this attenuation include metastatic malignancy, hepatic dysfunction of all severities, myocardial infarction, and neurologic disorders. This may be reflective of exacerbated or poorly controlled chronic conditions, such as 
hepatic dysfunction, in the setting of infection. Other complications, including myocardial infarction, have been shown to be strong predictors of short-term mortality in the setting of pneumonia, with increased risk of mortality still present at 1 year. ${ }^{6}$ As such, the attenuated but still present risk of mortality attributable to myocardial infarction in this study may reflect an overall poor prognosis of this group of patients.

\section{Limitations}

There were several limitations to this study. This study did not account for resistant $S$.

pneumoniae isolates or the impact of antibiotic treatment on 30-day mortality. However, a mortality rate of $14 \%$ for pneumonia and $24 \%$ for invasive disease in this study is similar to that reported for elderly and hospitalized individuals reported in the literature. ${ }^{1,2,6,7}$ As such, it is likely that the majority of patients in this cohort received appropriate antibiotic therapy. Also, the number of cases of pneumonia identified likely underestimates the actual number of cases, as the inclusion criteria for this study required both a diagnosis code and positive sputum culture. Next, although patients who received a pneumococcal vaccine within 5 years of infection were excluded, some patients may have been vaccinated prior to that 5-year period. Others have used this same definition as the impact of vaccination beyond 5 years in an elderly population is controversial, as the elderly exhibit decreased antibody activity over time against S. pneumoniae after vaccination. ${ }^{40,41}$ Further, it is possible that some patients did not report vaccinations they received outside of the Veterans Affairs healthcare system to their Veterans Affairs provider; however, this potential misclassification is likely to be low, as the vaccination rate in the overall Veterans Affairs cohort with pneumococcal infection was similar to a community population with serious pneumococcal infections (33\% vs 38\%). ${ }^{42}$ Extrapolation of these results to the general population is also limited, as the population primarily consisted of older white men. 
Furthermore, the Veteran population is generally considered to have poorer health status in comparison with the general population, which may also limit generalizability. ${ }^{43}$ Lastly, benefits from herd immunity through vaccination of children against pneumococcal disease could not be determined in this study.

\section{CONCLUSIONS}

This study identified independent predictors of mortality in a large cohort of unvaccinated Veterans with serious pneumococcal disease. These included several conditions that are already recognized by ACIP as recommendations for vaccination, including organ dysfunction and immunodeficiency. These results also suggest that individuals with neurologic disorders or dementia may also benefit from pneumococcal vaccination; however, further research is warranted. 


\section{ACKNOWLEDGMENTS}

The views expressed are those of the authors and do not necessarily reflect the position or policy of the U.S. Department of Veterans Affairs.

This work was supported, in part, by the Office of Research and Development, Department of Veterans Affairs. This study was sponsored, in part, by an Advancing Science through Pfizer Initiated Research grant from Pfizer Inc., as well as the Office of Academic Affiliations, Department of Veterans Affairs, and a Veterans Affairs New England Healthcare System Early Career Development Award.

IRB Number: 1172858

Article contents have been previously presented at the Making a Difference in Infectious Diseases annual meeting, 2016, Orlando Florida, and the 32nd Annual International Conference of Pharmacoepidemiology, August 2016, Dublin, Ireland.

Author contributions: conception and design of the study: JM, AC, HM, KL; data generation: JM, HM, AC; analysis and interpretation of the data: JM, AC, HM, KL; preparation or critical revision of the manuscript: JM, AC, HM, KL.

Kerry LaPlante has received research funding and/or served as an advisor or consultant for Merck (Cubist), BARD/Davol, Allergan (Forest Laboratories and Durata Therapeutics), Melinta 
Therapeutics, The Medicines Company, and Pfizer Inc. Aisling Caffrey has received research funding from Pfizer Inc. and Merck (Cubist). Haley Morrill has received research funding from Merck (Cubist) and is supported in part by a Veterans Affairs New England Career Development Award. No other financial disclosures were reported by the authors of this paper. 


\section{REFERENCES}

1. CDC. Active Bacterial Core Surveillance Report, Emerging Infections Program Network, Surveillance reports: Streptococcus pneumoniae; 20022013.http://www.cdc.gov/abcs/reports-findings/surv-reports.html. Accessed March 1, 2016.

2. Janoff EN, Musher DM. Streptococcus pneumoniae. In: Bennett JE, Dolin R, Blaser MJ, editors. Mandell, Douglas, and Bennett's Principles and Practice of Infectious Diseases. 8 ed: Elsevier; 2015:2310-2327.

3. CDC. Epidemiology and prevention of vaccine-preventable diseases. 12th ed. Washington, DC: Public Health Foundation; 2012.

4. Huang SS, Johnson KM, Ray GT, et al. Healthcare utilization and cost of pneumococcal disease in the United States. Vaccine. 2011;29(18):3398-3412. http://dx.doi.org/10.1016/j.vaccine.2011.02.088.

5. CDC. Pneumococcal Disease: Fast Facts. 2015. Www.cdc.gov/pneumococcal/about/facts.html. Accessed March 1, 2016.

6. Rae N, Finch S, Chalmers JD. Cardiovascular disease as a complication of community-acquired pneumonia. Curr Opin Pulm Med. 2016;22(3):212-218. http://dx.doi.org/10.1097/MCP.0000000000000261.

7. Musher DM, Alexandraki I, Graviss EA, et al. Bacteremic and nonbacteremic pneumococcal pneumonia. A prospective study. Medicine (Baltimore). 2000;79(4):210-221. http://dx.doi.org/10.1097/00005792-200007000-00002. 
8. CDC. Updated recommendations for prevention of invasive pneumococcal disease among adults using the 23-valent pneumococcal polysaccharide vaccine (PPSV23). MMWR Morb Mort Wkly Rep. 2010;59(34):1102-1106.

9. Marrie TJ, Tyrrell GJ, Garg S, Vanderkooi OG. Factors predicting mortality in invasive pneumococcal disease in adults in Alberta. Medicine (Baltimore). 2011;90(3):171-179. http://dx.doi.org/10.1097/MD.0b013e31821a5a76.

10. Veterans Health Administration. Where do I get the care I need? 2015. www.va.gov/health/FindCare.asp. Accessed June 3, 2015.

11. Morrill HJ, Caffrey AR, Noh E, LaPlante KL. Epidemiology of pneumococcal disease in a national cohort of older adults. Infect Dis Ther. 2014;3(1):19-33. http://dx.doi.org/10.1007/s40121-014-0025-y.

12. Price LE, Shea K, Gephart S. The Veteran's Affairs Corporate Data Warehouse. Nurs Admin Q. 2015;39(4):311-318. http://dx.doi.org/10.1097/NAQ.0000000000000118.

13. WHO. Pneumococcal vaccines WHO position paper. Wkly Epidemiol Rec. 2012;87(14):129-144.

14. Hosmer DW, Lemeshow S. Applied Logistic Regression. 2nd ed. New York: John Wiley \& Sons, Inc; 2005.

15. van de Beek D, de Gans J, Spanjaard L, Weisfelt M, Reitsma JB, Vermeulen M. Clinical features and prognostic factors in adults with bacterial meningitis. $N$ Engl $J$ Med. 2004;351(18):1849-1859. http://dx.doi.org/10.1056/NEJMoa040845.

16. Kalin M, Ortqvist A, Almela M, et al. Prospective study of prognostic factors in community-acquired bacteremic pneumococcal disease in 5 countries. J Infect Dis. 2000;182(3):840-847. http://dx.doi.org/10.1086/315760. 
17. Hanada S, Iwata S, Kishi K, et al. Host Factors and Biomarkers Associated with Poor Outcomes in Adults with Invasive Pneumococcal Disease. PLoS One. 2016;11(1):e0147877. http://dx.doi.org/10.1371/journal.pone.0147877.

18. Chi RC, Jackson LA, Neuzil KM. Characteristics and outcomes of older adults with community-acquired pneumococcal bacteremia. J Am Geriatr Soc. 2006;54(1):115120. http://dx.doi.org/10.1111/j.1532-5415.2005.00528.x.

19. Rudnick W, Liu Z, Shigayeva A, et al. Pneumococcal vaccination programs and the burden of invasive pneumococcal disease in Ontario, Canada, 1995-2011. Vaccine. 2013;31(49):5863-5871. http://dx.doi.org/10.1016/j.vaccine.2013.09.049.

20. Lin SH, Liao WH, Lai CC, et al. Comparison of clinical features, antimicrobial susceptibility, serotype distribution and outcomes of patients with hospital- and community-associated invasive pneumococcal disease. Int J Antimicrob Agents. 2010;36(2):119-123. http://dx.doi.org/10.1016/j.ijantimicag.2010.04.001.

21. Turett GS, Blum S, Fazal BA, Justman JE, Telzak EE. Penicillin resistance and other predictors of mortality in pneumococcal bacteremia in a population with high human immunodeficiency virus seroprevalence. Clin Infect Dis. 1999;29(2):321-327. http://dx.doi.org/10.1086/520209.

22. Kumashi P, Girgawy E, Tarrand JJ, Rolston KV, Raad, II, Safdar A. Streptococcus pneumoniae bacteremia in patients with cancer: disease characteristics and outcomes in the era of escalating drug resistance (1998-2002). Medicine (Baltimore). 2005;84(5):303-312. http://dx.doi.org/10.1097/01.md.0000180045.26909.29. 
23. Shigayeva A, Rudnick W, Green K, et al. Invasive Pneumococcal Disease Among Immunocompromised Persons: Implications for Vaccination Programs. Clin Infect Dis. 2016;62(2):139-147. http://dx.doi.org/10.1093/cid/civ803.

24. Kyaw MH, Rose CE, Jr., Fry AM, et al. The influence of chronic illnesses on the incidence of invasive pneumococcal disease in adults. J Infect Dis. 2005;192(3):377386. http://dx.doi.org/10.1086/431521.

25. Wagner C, Popp W, Posch M, Vlasich C, Rosenberger-Spitzy A. Impact of pneumococcal vaccination on morbidity and mortality of geriatric patients: a casecontrolled study. Gerontology. 2003;49(4):246-250. http://dx.doi.org/10.1159/000070405.

26. Becker-Dreps S, Amaya E, Liu L, et al. Impact of a combined pediatric and adult pneumococcal immunization program on adult pneumonia incidence and mortality in Nicaragua. Vaccine. 2015;33(1):222-227. http://dx.doi.org/10.1016/j.vaccine.2014.10.073.

27. Vila-Corcoles A, Ochoa-Gondar O, Hospital I, et al. Protective effects of the 23valent pneumococcal polysaccharide vaccine in the elderly population: the EVAN-65 study. Clin Infect Dis. 2006;43(7):860-868. http://dx.doi.org/10.1086/507340.

28. Office of Disease Prevention and Health Promotion. Healthy People 2020. Immunization and Infectious Diseases. 2016. www.healthypeople.gov/2020/topicsobjectives/topic/immunization-and-infectious-diseases/objectives\#4658. Accessed February 24, 2016. 
29. CDC. National Health Interview Survey. 2013.

http://www.cdc.gov/nchs/nhis/nhis_2013_data_release.htm. Accessed February 24, 2016.

30. Robinson KA, Baughman W, Rothrock G, et al. Epidemiology of invasive Streptococcus pneumoniae infections in the United States, 1995-1998: Opportunities for prevention in the conjugate vaccine era. JAMA. 2001;285(13):1729-1735. http://dx.doi.org/10.1001/jama.285.13.1729.

31. CDC. Use of 13-Valent pneumococcal conjugate vaccine and 23-valent pneumococcal polysaccharide vaccine for adults with immunocompromising conditions: recommendations of the Advisory Committee on Immunization Practices (ACIP). MMWR Morb Mort Wkly Rep. 2012;61(40):816-819.

32. Pilishvili T, Bennett NM. Pneumococcal Disease Prevention Among Adults: Strategies for the Use of Pneumococcal Vaccines. Am J Prev Med. 2015;49(6 Suppl 4):S383-390. http://dx.doi.org/10.1016/j.amepre.2015.09.008.

33. Isturiz R, Webber C. Prevention of adult pneumococcal pneumonia with the 13-valent pneumococcal conjugate vaccine: CAPiTA, the community-acquired pneumonia immunization trial in adults. Hum Vaccin Immunother. 2015;11(7):1825-1827. http://dx.doi.org/10.1080/21645515.2015.1043502.

34. Torres A, Peetermans WE, Viegi G, Blasi F. Risk factors for community-acquired pneumonia in adults in Europe: a literature review. Thorax. 2013;68(11):1057-1065. http://dx.doi.org/10.1136/thoraxjnl-2013-204282. 
35. Smith GL, Masoudi FA, Shlipak MG, Krumholz HM, Parikh CR. Renal impairment predicts long-term mortality risk after acute myocardial infarction. J Am Soc Nephrol. 2008;19(1):141-150. http://dx.doi.org/10.1681/ASN.2007050554.

36. Cardarelli F, Bellasi A, Ou FS, et al. Combined impact of age and estimated glomerular filtration rate on in-hospital mortality after percutaneous coronary intervention for acute myocardial infarction (from the American College of Cardiology National Cardiovascular Data Registry). Am J Cardiol. 2009;103(6):766771. http://dx.doi.org/10.1016/j.amjcard.2008.11.033.

37. Leijon S, Bergh I, Terstappen K. Pressure ulcer prevalence, use of preventive measures, and mortality risk in an acute care population: a quality improvement project. J Wound Ostomy Continence Nurs. 2013;40(5):469-474. http://dx.doi.org/10.1097/WON.0b013e3182a22032.

38. Ishiguro T, Takayanagi N, Yamaguchi S, et al. Etiology and factors contributing to the severity and mortality of community-acquired pneumonia. Intern Med. 2013;52(3):317-324. http://dx.doi.org/10.2169/internalmedicine.52.8830.

39. Alzheimer's Association. 2014 Alzheimer's Disease: Facts and Figures. 2014. https://www.alz.org/downloads/facts_figures_2014.pdf. Accessed April 13, 2016.

40. Romero-Steiner S, Musher DM, Cetron MS, et al. Reduction in functional antibody activity against Streptococcus pneumoniae in vaccinated elderly individuals highly correlates with decreased IgG antibody avidity. Clin Infect Dis. 1999;29(2):281-288. http://dx.doi.org/10.1086/520200.

41. Vila-Corcoles A, Ochoa-Gondear O, Rodriguez-Blanco T, et al. Clinical effectiveness of pneumococcal vaccination against acute myocardial infarction and stroke in people 
over 60 years: the CAMPAMIS study, one-year follow-up. BMC Public Health. 2012;12:222. http://dx.doi.org/10.1186/1471-2458-12-222.

42. Vila-Corcoles A, Ochoa-Gondar O, Guzman A, Rodriguez-Blanco T, Salsench E, Fuentes CM. Effectiveness of the 23-valent polysaccharide pneumococcal vaccine against invasive pneumococcal disease in people 60 years and older. BMC Infect Dis. 2010;10:73. http://dx.doi.org/10.1186/1471-2334-10-73.

43. Agha Z, Lofgren R, VanRuiswyk J, Layde P. Are patients at Veterans Affairs medical centers sicker? A comparative analysis of health status and medical resource use.

Arch Intern Med. 2000;160(21):3252-3257.

http://dx.doi.org/10.1001/archinte.160.21.3252. 
Table 1. Population Demographics and Characteristics of Hospitalized, Unvaccinated Patients With Severe Pneumococcal Infections

\begin{tabular}{|c|c|c|c|}
\hline Demographic/Characteristic & $\begin{array}{l}\text { Alive at } 30 \text { days } \\
\quad(n=7,966)\end{array}$ & $\begin{array}{c}\text { Died within } 30 \\
\text { days } \\
(\mathrm{n}=1,764)\end{array}$ & $p$-value ${ }^{a}$ \\
\hline Age (years), mean (SD) & $67( \pm 11)$ & $71( \pm 11)$ & $<0.01$ \\
\hline Male gender & 7,795 (97.9) & 1,740 (98.6) & 0.03 \\
\hline \multicolumn{4}{|l|}{ Race } \\
\hline American Indian & $98(1.2)$ & $14(0.8)$ & 0.12 \\
\hline Asian or Pacific Islander & $63(0.8)$ & $11(0.6)$ & 0.46 \\
\hline Black & $1,054(13.2)$ & $238(13.5)$ & 0.77 \\
\hline White & $6,297(79.0)$ & $1,354(76.8)$ & 0.03 \\
\hline Unknown & $454(5.7)$ & $147(8.3)$ & $<0.01$ \\
\hline \multicolumn{4}{|l|}{ Region of facility } \\
\hline Midwest & $1,996(25.1)$ & $382(21.7)$ & $<0.01$ \\
\hline Northeast & $1,147(14.4)$ & $290(16.4)$ & 0.03 \\
\hline South & $2,766(34.7)$ & $695(39.4)$ & $<0.01$ \\
\hline West & $2,057(25.8)$ & $397(22.5)$ & $<0.01$ \\
\hline \multicolumn{4}{|l|}{ Treating specialty } \\
\hline Intensive care unit & $1,830(23.0)$ & $699(39.6)$ & $<0.01$ \\
\hline \multicolumn{4}{|l|}{ Current pneumococcal infection } \\
\hline Pneumonia & $5,204(65.3)$ & $871(49.4)$ & $<0.01$ \\
\hline Bacteremia & $1,969(24.7)$ & $585(33.2)$ & $<0.01$ \\
\hline Pneumonia and bacteremia & $755(9.5)$ & $305(17.3)$ & $<0.01$ \\
\hline Invasive pneumococcal infection ${ }^{\mathrm{b}}$ & $2,762(34.7)$ & $893(50.6)$ & $<0.01$ \\
\hline \multicolumn{4}{|l|}{ Other infections during admission ${ }^{c}$} \\
\hline Infective arthritis & $41(0.5)$ & $<5$ & 0.02 \\
\hline Infective endocarditis & $27(0.3)$ & $14(0.8)$ & $<0.01$ \\
\hline Skin or soft tissue infection & $336(4.2)$ & $114(6.5)$ & $<0.01$ \\
\hline Urinary tract infection & $371(4.7)$ & $133(7.5)$ & $<0.01$ \\
\hline \multicolumn{4}{|l|}{ Other positive cultures during admission } \\
\hline E. coli & $61(0.8)$ & $17(1.0)$ & 0.4 \\
\hline Gram-negative organisms & $149(1.9)$ & $60(3.4)$ & $<0.01$ \\
\hline MRSA & $106(1.3)$ & $29(1.6)$ & 0.31 \\
\hline MSSA & $139(1.7)$ & $57(3.2)$ & $<0.01$ \\
\hline Pseudomonas spp. & $104(1.3)$ & $37(2.1)$ & 0.01 \\
\hline Vancomycin-resistant Enterococcus spp. & $6(0.1)$ & $7(0.4)$ & $<0.01$ \\
\hline \multicolumn{4}{|l|}{ Comorbidities during admission } \\
\hline Alcohol abuse & $734(9.2)$ & $147(8.3)$ & 0.24 \\
\hline Amputation & $52(0.7)$ & $5(0.3)$ & 0.07 \\
\hline Cardiac arrhythmia & $1,496(18.8)$ & $503(28.5)$ & $<0.01$ \\
\hline Cerebrovascular disease & $317(4.0)$ & $124(7.0)$ & $<0.01$ \\
\hline Chronic renal disease & $584(7.3)$ & $195(11.1)$ & $<0.01$ \\
\hline
\end{tabular}


Chronic respiratory disease

Coagulopathy

Coronary artery disease

Dementia

Diabetes mellitus

Dialysis

Drug abuse

Fluid or electrolyte disorders

Heart failure

Hepatic disease, mild

Hepatic disease, moderate or severe

Hypertension

Metastatic malignancy

Neurological disorders

Neutropenia

Obesity

Chronic pressure ulcer

Infection previous year

Bacteremia $^{c}$

Pneumococcal infection ${ }^{d}$

Pneumonia $^{c}$

Skin or soft tissue infection ${ }^{c}$

Urinary tract infection ${ }^{\mathrm{c}}$

Positive cultures within previous year

E. coli

Gram-negative organisms

MRSA

MSSA

Pseudomonas spp.

Vancomycin-resistant Enterococcus spp.

Medical history

Alcohol abuse

Amputation

Cardiac arrhythmia

Cerebrovascular disease

Chronic renal disease

Chronic respiratory disease

Coagulopathy

Coronary artery disease

Dementia

Diabetes mellitus

Dialysis

$$
\begin{gathered}
3,106(39.0) \\
267(3.4) \\
1,370(17.2) \\
109(1.4) \\
1,256(15.8) \\
150(1.9) \\
340(4.3) \\
1,649(20.7) \\
1,040(13.1) \\
404(5.1) \\
64(0.8) \\
2,544(31.9) \\
227(2.8) \\
326(4.1) \\
23(0.3)
\end{gathered}
$$

1,894 (23.8)

197 (2.5)

217 (2.7)

2,256 (28.3)

1,911 (24.0)

856 (10.7)

$583(7.3)$

$78(1.0)$

$141(1.8)$

$122(1.5)$

$201(2.5)$

127 (1.6)

$6(0.1)$

1,261 (15.8)

114 (1.4)

1,707 (21.4)

770 (9.7)

$668(8.4)$

3,609 (45.3)

359 (4.5)

$1,999(25.1)$

$182(2.3)$

1,709 (21.5)

$66(0.8)$
579 (32.8)

$138(7.8)$

$336(19.0)$

$59(3.3)$

252 (14.3)

$104(5.9)$

$30(1.7)$

$455(25.8)$

334 (18.9)

140 (7.9)

57 (3.2)

453 (25.7)

$158(9.0)$

$133(7.5)$

$23(1.3)$

292 (16.6)

92 (5.2)

109 (6.2)

686 (38.9)

625 (35.4)

281 (15.9)

266 (15.1)

43 (2.4)

$71(4.0)$

67 (3.8)

109 (6.2)

$53(3.0)$

7 (0.4)

313 (17.7)

39 (2.2)

592 (33.6)

301 (17.1)

266 (15.1)

911 (51.6)

184 (10.4)

$611(34.6)$

113 (6.4)

476 (27.0)

33 (1.9)
$<0.01$

$<0.01$

0.06

$<0.01$

0.12

$<0.01$

$<0.01$

$<0.01$

$<0.01$

$<0.01$

$<0.01$

$<0.01$

$<0.01$

$<0.01$

$<0.01$

$<0.01$

$<0.01$

$<0.01$

$<0.01$

$<0.01$

$<0.01$

$<0.01$

$<0.01$

$<0.01$

$<0.01$

$<0.01$

$<0.01$

0.51

0.05

0.02

$<0.01$

$<0.01$

$<0.01$

$<0.01$

$<0.01$

$<0.01$

$<0.01$

$<0.01$

$<0.01$ 
Drug abuse

$636(8.0)$
$1,566(19.7)$
$1,324(16.6)$
$590(7.4)$
$115(1.4)$
$3,952(49.6)$
$2,535(31.8)$
$382(4.8)$
$522(6.6)$
$28(0.4)$
$334(4.2)$

$118(6.7)$

0.07

Fluid or electrolyte disorders

$625(35.4)$

$<0.01$

Heart failure

$489(27.7)$

$<0.01$

Hepatic disease, mild

$241(13.7)$

$<0.01$

Hepatic disease, moderate or severe

$79(4.5)$

$<0.01$

Hypertension

$1,035(58.7)$

$<0.01$

Immunocomproming condition ${ }^{\mathrm{e}}$

Metastatic malignancy

Neurological disorders

$747(42.3)$

$<0.01$

$239(13.6)$

$<0.01$

$220(12.5)$

$<0.01$

Neutropenia

$334(4.2)$

$19(1.1)$

$<0.01$

Pressure ulcer

175 (9.9)

$<0.01$

Receiving immunocompromising

medication $^{\mathrm{f}}$

$1,795(22.5)$

$498(28.2)$

$<0.01$

Cigarette smoking

$3,777(47.4)$

$674(38.2)$

$<0.01$

$3(1-5)$

$4(3-6)$

$<0.01$

Length of stay (days), median (IQR)

$8(3-15)$

$<0.01$

Note: Boldface indicates statistical significance $(p<0.05)$.

${ }^{\text {a }}$ Chi-square or Fisher's Exact test, as appropriate.

${ }^{\mathrm{b}}$ Includes bacteremia, pneumonia and bacteremia, meningitis, bacteremia and meningitis, and pneumonia, bacteremia, and meningitis

${ }^{c}$ Attributed to any organism.

${ }^{\mathrm{d}}$ Includes all infection types from any positive Streptococcus pneumoniae culture site.

${ }^{\mathrm{e}} \mathrm{HIV}$, AIDS-defining illness, solid malignancy, or hematologic malignancy within 1 year of positive $S$. pneumoniae culture.

${ }^{\mathrm{f}}$ Received corticosteroid, monoclonal antibody, or antineoplastic agent within 30 days of positive Streptococcus pneumoniae culture

IQR, interquartile range; MRSA, methicillin-resistant Staphylococcus aureus; MSSA, methicillin-sensitive Staphylococcus aureus; spp., species 
Table 2. Predictors of 30-Day All-Cause Mortality in Unvaccinated Patients With Severe Pneumococcal Disease

\begin{tabular}{|c|c|c|c|c|}
\hline \multirow[b]{2}{*}{ Predictor } & \multicolumn{2}{|c|}{ Predictors during hospitalization } & \multicolumn{2}{|c|}{ Predictors within 1 year } \\
\hline & $\begin{array}{c}\text { Unadjusted OR } \\
(95 \% \mathrm{CI})\end{array}$ & $\begin{array}{c}\text { Adjusted OR } \\
(95 \% \mathrm{CI})\end{array}$ & $\begin{array}{c}\text { Unadjusted OR } \\
(95 \% \mathrm{CI})\end{array}$ & $\begin{array}{c}\text { Adjusted OR } \\
(95 \% \mathrm{CI})\end{array}$ \\
\hline \multicolumn{5}{|c|}{ Pneumococcal vaccine currently recommended } \\
\hline Age & $1.03(1.02-1.03)$ & $1.03(1.03-1.04)$ & - & - \\
\hline Congestive heart failure & $1.56(1.36-1.78)$ & $1.50(1.25-1.81)$ & - & - \\
\hline $\begin{array}{l}\text { Corticosteroid use within } 30 \\
\text { days of culture }\end{array}$ & 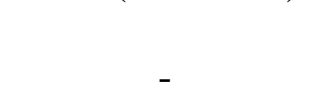 & 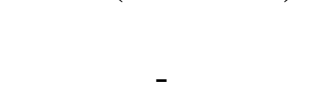 & $1.27(1.12-1.43)$ & $1.3(1.13-1.50)$ \\
\hline Dialysis & $3.27(2.53-4.22)$ & $3.35(2.37-4.72)$ & - & - \\
\hline Metastatic malignancy & $3.35(2.72-4.14)$ & $2.54(1.89-3.41)$ & $3.11(2.62-3.69)$ & $1.53(1.19-1.97)$ \\
\hline Mild liver disease & $1.61(1.32-1.97)$ & $1.63(1.20-2.22)$ & $1.98(1.69-2.32)$ & $1.35(1.07-1.71)$ \\
\hline Moderate or severe liver disease & $4.12(2.87-5.91)$ & $2.47(1.53-3.99)$ & $3.20(2.39-4.28)$ & $1.49(1.01-2.20)$ \\
\hline Myocardial Infarction & $2.08(1.67-2.58)$ & $1.75(1.35-2.27)$ & $2.08(1.74-2.48)$ & $1.31(1.06-1.62)$ \\
\hline Neutropenia $^{\mathrm{a}}$ & $4.56(2.55-8.15)$ & $2.67(1.32-5.42)$ & - & - \\
\hline Non-metastatic malignancy & $2.21(1.95-2.50)$ & $2.34(1.91-2.87)$ & - & - \\
\hline \multicolumn{5}{|c|}{ Pneumococcal vaccine not currently recommended } \\
\hline Cardiac arrhythmia & $1.73(1.53-1.94)$ & $1.41(1.21-1.63)$ & - & - \\
\hline Cerebrovascular disease & - & - & $1.92(1.66-2.22)$ & $1.25(1.05-1.49)$ \\
\hline Chronic pressure ulcers & $2.17(1.69-2.80)$ & $1.42(1.04-1.92)$ & $2.15(2.08-3.05)$ & $2.04(1.48-2.82)$ \\
\hline Coagulopathy & $2.45(1.98-3.03)$ & $2.3(1.75-3.01)$ & - & - \\
\hline Dementia & $2.49(1.81-3.44)$ & $1.80(1.23-2.61)$ & - & - \\
\hline Elixhauser comorbidity score & - & - & $1.19(1.17-1.21)$ & $1.16(1.12-1.20)$ \\
\hline Endocarditis & $2.35(1.23-4.50)$ & $2.74(1.28-5.90)$ & - & - \\
\hline Fluid or electrolyte disorders & $1.33(1.18-1.50)$ & $1.69(1.43-1.99)$ & $2.24(2.01-2.51)$ & $1.20(1.03-1.39)$ \\
\hline Gram-negative infection & - & - & $2.33(1.74-3.11)$ & $1.48(1.05-2.07)$ \\
\hline MRSA infection & - & - & $2.54(1.88-3.44)$ & $1.59(1.12-2.25)$ \\
\hline Neurological disorders & $1.91(1.55-2.35)$ & $1.86(1.42-2.45)$ & $2.03(1.72-2.40)$ & $1.28(1.02-1.59)$ \\
\hline Vancomycin-resistant & & & & \\
\hline Enterococcus spp. infection & $5.28(1.77-15.74)$ & $3.78(1.02-14.17)$ & - & - \\
\hline Weight loss & - & - & $2.06(1.79-2.37)$ & $1.21(1.02-1.44)$ \\
\hline
\end{tabular}


Other predictors

Hospital admission in

northeastern U.S.

$1.31(1.11-1.55) \quad 1.29(1.06-1.56)$

Hospital admission in southern

U.S.

ICU level of care

$1.30(1.14-1.49)$

$1.33(1.14-1.56)$

Invasive pneumococcal disease

$2.20(1.97-2.45) \quad 2.26(1.99-2.57)$

$1.93(1.74-2.14) \quad 2.1(1.85-2.39)$

Notes: Final multivariable logistic regression model included 54 variables (194 variables assessed). Significant variables during admission in the final model $(\mathrm{OR}<1)$ : length of stay, Elixhauser comorbidity score, hypertension, streptococcal spp. infection, amputation, infective arthritis. Significant variables within previous year in the final model (OR $<1)$ : history of peripheral vascular disease, cigarette smoking, drug abuse, dialysis. Other variables included in the final model (OR<1): obesity, race, hospital admission in the midwestern and western U.S. (reference).

${ }^{a}$ Neutropenia may be classified as an immunocompromising condition, which was a recommendation for PPSV-23 vaccination in 1984.

Adjusted, results of multivariable logistic regression; ICU, intensive care unit; MRSA, methicillin-resistant Staphylococcus aureus; Unadjusted, results of univariable logistic regression 